\title{
Numerical blow-up for nonlinear diffusion equation with neumann boundary conditions
}

\author{
Ardjouma Ganon ${ }^{\mathrm{a}, *}$, Manin Mathurin Taha ${ }^{\mathrm{a}}$, N'guessan Koffi ${ }^{\mathrm{b}}$, Augustin Kidjégbo Touréa \\ a Institut National Polytechnique Felix Houphouët-Boigny Yamoussoukro, BP 2444, Cöte d'Ivoire. \\ ${ }^{b}$ UFR SED, Université Alassane Ouattara de Bouaké, 01 BP V 18 Bouaké 01, Cöte d'lvoire.
}

\begin{abstract}
This work is concerned with the study of the numerical approximation for the nonlinear diffusion equation $\left(\mathrm{u}^{\mathrm{m}}\right)_{\mathrm{t}}=$ $u_{x x}, 0<x<1, t>0$, under Neumann boundary conditions $u_{x}(0, t)=0, u_{x}(1, t)=u^{\alpha}(1, t)$, $t>0$. First, we obtain a semidiscrete scheme by the finite differences method and prove the convergence of its solution to the continuous one. Then, we establish the numerical blow-up and the convergence of the numerical blow-up time to the theoretical one when the mesh size goes to zero. Finally, we illustrate our analysis with some numerical experiments.
\end{abstract}

Keywords: Nonlinear diffusion equation, numerical blow-up, finite differences, arc length transformation, Aitken $\Delta^{2}$ method. 2020 MSC: 35B44, 35B51, 35K20, 65M06.

(C)2021 All rights reserved.

\section{Introduction}

Consider the following nonlinear diffusion equation with neumann boundary conditions

$$
\begin{cases}\left(u^{m}\right)_{t}=u_{x x}, & 0<x<1, t>0 \\ u_{x}(0, t)=0, u_{x}(1, t)=u^{\alpha}(1, t), & t>0 \\ u(x, 0)=u_{0}(x) \geqslant \delta>0, & 0 \leqslant x \leqslant 1\end{cases}
$$

where $m>0, \alpha<\infty$ and $u_{0} \in C^{2+v}([0,1])$ for some $0<v<1$, and verifies the compatibility conditions $u_{0}^{\prime}(0)=0$ and $u_{0}^{\prime}(1)=u_{0}^{\alpha}(1)$. There are many evolution equations whose solutions develop singularity in finite time, namely $\mathrm{T}$. Close to this finite time $\mathrm{T}$, solutions become unbounded at certain points of the space. This phenomenon is called blow-up, and $T$ is called the blow-up time. The set of points where blow-up occurs is called the blow-up set. The blow-up phenomenon has been the focus of many authors in recent years. Some were interested in the theoretical analysis $[2,5,6,10]$, and others in the numerical one $[1,3,4,7,8,11]$. Existence and uniqueness of regular solution, and the following results have been proved by Filo in [6]:

\footnotetext{
*Corresponding author

Email addresses: ardjganon@gmail.com (Ardjouma Ganon), tahammath@gmail.com (Manin Mathurin Taha), nkrasoft@yahoo.fr (N'guessan Koffi), latoureci@gmail.com (Augustin Kidjégbo Touré)
}

doi: $10.22436 /$ jnsa.014.02.03

Received: 2020-02-25 Revised: 2020-03-03 Accepted: 2020-04-12 
If $0<m<\alpha \leqslant 1$, the solution of (1.1) blows up in finite time $T$ on the whole interval [0,1], but if $0<\mathrm{m}<1<\alpha$, blow-up occurs only at the boundary $x=1$. And if $m>1$ with $2 \alpha>m+1$, every solution must blow up in a finite time. Furthermore, if $\alpha>m>1$, the solution of (1.1) blows up at $x=1$. He also established blow-up rate for some cases. Later, Dend and $\mathrm{Xu}$ [2] completed the blow-up rate results of the solution of (1.1).

This paper deals with the numerical approximations of (1.1). Our goal is to prove the blow-up of the numerical solution and the convergence of the numerical blow-up time without putting strong assumption on initial data (we only use assumptions under which the solution of the continuous problem blows up). Problem (1.1) can be rewritten in the following form

$$
\begin{cases}u_{t}=\frac{1}{m} u^{1-} u_{x x}, & (x, t) \in(0,1) \times(0, T), \\ u_{x}(0, t)=0, u_{x}(1, t)=u^{\alpha}(1, t), & t \in(0, T), \\ u(x, 0)=u_{0}(x), & x \in[0,1],\end{cases}
$$

The rest of the work is organized as follows. In the next section, we present a semidiscrete scheme of the problem (1.1). In Section 3, we give some properties of this semidiscrete scheme. In Section 4, under some conditions, we prove that the solution of the semidiscrete scheme of (1.1) blows up in finite time and that the numerical blow-up time converges to the theoretical one when the mesh size goes to zero. Finally, in the last section, we illustrate our analysis by giving some numerical results.

\section{Semidiscrete problem}

Let $I$ be a positive integer and define the grid $x_{i}=i h, i=0, \ldots, I$, where $h=\frac{1}{I}$ is the mesh parameter. We approximate the solution $u$ of the problem (1.2) by the solution $U_{h}(t)=\left(U_{0}(t), \ldots, U_{I}(t)\right)^{\top}$ of the following semidiscrete scheme

$$
\begin{aligned}
\frac{d U_{i}(t)}{d t} & =\frac{1}{m} u_{i}^{1-m}(t) \delta^{2} u_{i}(t), \quad i=0, \ldots, I-1, \quad t \in\left(0, T_{h}\right), \\
\frac{d U_{I}(t)}{d t} & =\frac{1}{m} u_{I}^{1-m}(t)\left(\delta^{2} u_{I}(t)+\frac{2}{h} u_{I}^{\alpha}(t)\right), \quad t \in\left(0, T_{h}\right), \\
u_{i}(0) & =\varphi_{i}>0, \quad i=0, \ldots, I,
\end{aligned}
$$

where for $t \in\left(0, T_{h}\right)$,

$$
\begin{aligned}
& \delta^{2} u_{i}(t)=\frac{U_{i+1}(t)-2 U_{i}(t)+U_{I-1}(t)}{h^{2}}, \quad i=1, \ldots, I-1, \\
& \delta^{2} u_{0}(t)=\frac{2 U_{1}(t)-2 U_{0}(t)}{h^{2}}, \quad \delta^{2} u_{I}(t)=\frac{2 U_{I-1}(t)-2 U_{I}(t)}{h^{2}},
\end{aligned}
$$

and $\left[0, T_{h}\right)$, the maximal time interval on which $\left\|U_{h}(t)\right\|_{\infty}$ is finite, with $\left\|U_{h}(t)\right\|_{\infty}=\max _{0 \leqslant i \leqslant I}\left|U_{i}(t)\right|$. When $T_{h}$ is finite, we say that the solution $U_{h}(t)$ blows up in finite time and the time $T_{h}$ is called the blow-up time of the solution $\mathrm{U}_{\mathrm{h}}(\mathrm{t})$. Denote

$$
\delta_{*}^{2} u_{i}(t)= \begin{cases}\delta^{2} u_{i}(t), & \text { if } i=0, \ldots, I-1, \\ \delta^{2} U_{I}(t)+\frac{2}{h} u_{I}^{\alpha}(t), & \text { if } i=I .\end{cases}
$$

\section{Properties of the semidiscrete scheme}

We give in this section some important results which will be used later. 
Lemma 3.1. Let $f \in C^{0}(\mathbb{R}, \mathbb{R})$, if $V_{h}, W_{h} \in C^{1}\left([0, T), \mathbb{R}^{I+1}\right)$ and $a_{h} \in C^{0}\left([0, T), \mathbb{R}_{+}^{I+1}\right)$ are such that for $i=0, \ldots, \mathrm{I}$

$$
\begin{aligned}
\frac{d V_{i}(t)}{d t}-a_{i}(t) \delta^{2} V_{i}(t)-f\left(V_{i}(t)\right) & <\frac{d W_{i}(t)}{d t}-a_{i}(t) \delta^{2} W_{i}(t)-f\left(W_{i}(t)\right), t \in(0, T), \\
V_{i}(0) & <W_{i}(0), \quad i=0, \ldots, I,
\end{aligned}
$$

then we have $\mathrm{V}_{\mathrm{i}}(\mathrm{t})<\mathrm{W}_{\mathrm{i}}(\mathrm{t}), \quad 0 \leqslant i \leqslant \mathrm{I}, \quad \mathrm{t} \in(0, \mathrm{~T})$.

Proof. Let us define the vector $Z_{h}(t)=W_{h}(t)-V_{h}(t)$. Let $t_{0}$ be the first $t \in(0, T)$ such that $Z_{i}(t)>0$ for $t \in\left[0, t_{0}\right), 0 \leqslant i \leqslant I$, but $Z_{i_{0}}\left(t_{0}\right)=0$ for a certain $i_{0} \in\{0, \ldots, I\}$. It is easy to see that

$$
\frac{d}{d t} Z_{i_{0}}\left(t_{0}\right)=\lim _{k \rightarrow 0} \frac{Z_{i_{0}}\left(t_{0}\right)-Z_{i_{0}}\left(t_{0}-k\right)}{k} \leqslant 0, \quad \delta^{2} Z_{i_{0}}\left(t_{0}\right) \geqslant 0,
$$

which implies that

$$
\frac{d}{d t} Z_{i_{0}}\left(t_{0}\right)-a_{i_{0}}(t) \delta^{2} Z_{i_{0}}\left(t_{0}\right)+f\left(W_{i_{0}}\left(t_{0}\right)\right)-f\left(V_{i_{0}}\left(t_{0}\right)\right) \leqslant 0,
$$

but this inequality contradicts (3.1) and the proof is complete.

Lemma 3.2. Let $\mathrm{U}_{\mathrm{h}}$ be a solution of (2.1)-(2.3), then

$$
\mathrm{U}_{\mathrm{i}}(\mathrm{t})>0, \quad i=0, \ldots, \mathrm{I}, \mathrm{t} \in\left(0, \mathrm{~T}_{\mathrm{h}}\right) .
$$

Proof. The semidiscret scheme (2.1)-(2.3) can be rewritten as follows

$$
\begin{aligned}
\frac{d\left(U_{i}^{m}(t)\right)}{d t} & =\delta^{2} U_{i}(t), \quad i=0, \ldots, I-1, \quad t \in\left(0, T_{h}\right), \\
\frac{d\left(U_{I}^{m}(t)\right)}{d t} & =\delta^{2} U_{I}(t)+\frac{2}{h} U_{I}^{\alpha}(t), \quad t \in\left(0, T_{h}\right), \\
U_{i}(0) & =\varphi_{i}>0, \quad i=0, \ldots, I .
\end{aligned}
$$

Let $t_{0}$ be the first $t \in\left(0, T_{h}\right)$ such that $U_{i}(t)>0$ for $t \in\left[0, t_{0}\right), 0 \leqslant i \leqslant I$, but $U_{i_{0}}\left(t_{0}\right)=0$ for a certain $\mathfrak{i}_{0} \in\{0, \ldots, \mathrm{I}\}$. One can easily check that

$$
\frac{d\left(u_{i_{0}}^{m}\left(t_{0}\right)\right)}{d t}=\lim _{\epsilon \rightarrow 0} \frac{u_{i_{0}}^{m}\left(t_{0}\right)-u_{i_{0}}^{m}\left(t_{0}-\epsilon\right)}{\epsilon} \leqslant 0, \quad \delta^{2} u_{i_{0}}\left(t_{0}\right)>0,
$$

which implies that

$$
\begin{aligned}
& \frac{d\left(u_{i_{0}}^{m}\left(t_{0}\right)\right)}{d t}-\delta^{2} u_{i_{0}}\left(t_{0}\right)<0 \quad \text { if } 0 \leqslant i_{0} \leqslant I-1, \\
& \frac{d\left(u_{I}^{m}\left(t_{0}\right)\right)}{d t}-\delta^{2} u_{I}\left(t_{0}\right)-\frac{2}{h} u_{I}^{\alpha}\left(t_{0}\right)<0 \quad \text { if } i_{0}=I .
\end{aligned}
$$

But these inequalities contradict (3.2)-(3.3) and we get the expected result.

Lemma 3.3. Let $\mathrm{U}_{\mathrm{h}}$ be a solution of (2.1)-(2.3) and the initial condition at (2.3) verifies $\delta_{*}^{2} \varphi_{i} \geqslant 0,0 \leqslant i \leqslant \mathrm{I}$. Then, $\frac{d U_{i}(t)}{d t} \geqslant 0$ for $0 \leqslant i \leqslant I, t \in\left[0, T_{h}\right)$.

Proof. Take $T_{0}<T_{h}$ fixed. Let us define the vector $W_{h}(t)$ such that $W_{i}(t)=\frac{d}{d t} U_{i}(t)$ for $0 \leqslant i \leqslant I, t \in$ $\left[0, \mathrm{~T}_{0}\right]$. We have

$$
\frac{d W_{i}(t)}{d t}=\frac{1}{m} u_{i}^{1-m}(t) \delta^{2} W_{i}(t)+\frac{1-m}{m} u_{i}^{-m}(t) \delta^{2} u_{i}(t) W_{i}(t), \quad 0 \leqslant i \leqslant I-1,
$$




$$
\frac{d W_{I}(t)}{d t}=\frac{1}{m} u_{I}^{1-m}(t) \delta^{2} W_{I}(t)+\left(\frac{1-m}{m} u_{I}^{-m}(t) \delta^{2} U_{I}(t)+\frac{2(1-m+\alpha)}{m h} U_{I}^{\alpha-m}(t)\right) W_{I}(t) .
$$

Let $\mathrm{K}$ be a positive constant satisfying

and

$$
K>\max _{0 \leqslant i \leqslant I, 0 \leqslant t \leqslant T_{0}}\left\{\left|\frac{1-m}{m} u_{i}^{-m}(t) \delta^{2} u_{i}(t)\right|\right\}
$$

$$
K>\max _{0 \leqslant t \leqslant T_{0}}\left\{\left|\frac{1-m}{m} U_{I}^{-m}(t) \delta^{2} U_{I}(t)\right|+\frac{2|1-m+\alpha|}{m h} u_{I}^{\alpha-m}(t)\right\} .
$$

Consider the vector $Z_{h}(t)=W_{h}(t) e^{-K t}$. It is clear that $Z_{h}(0) \geqslant 0$ since $W_{h}(0) \geqslant 0$. Let $t_{0}$ be the first $t \in\left(0, T_{0}\right]$ such that $Z_{i}(t) \geqslant 0$ for $t \in\left[0, t_{0}\right)$, but $Z_{i_{0}}\left(t_{0}\right)<0$ for a certain $i_{0} \in\{0, \ldots, I\}$. Without lost of generality, we may suppose that $\mathfrak{i}_{0}$ is the smallest integer which satisfies the above inequality. Then we have

$$
\begin{aligned}
\frac{d}{d t} Z_{\mathfrak{i}_{0}}\left(t_{0}\right) & =\lim _{k \rightarrow 0} \frac{Z_{i_{0}}\left(t_{0}\right)-Z_{i_{0}}\left(t_{0}-k\right)}{k} \leqslant 0, \\
\delta^{2} Z_{\mathfrak{i}_{0}}\left(t_{0}\right) & >0 .
\end{aligned}
$$

From relations (3.5)-(3.6) and (3.8), we can easily show that

$$
\begin{aligned}
\frac{d Z_{i_{0}}\left(t_{0}\right)}{d t}>\left(\frac{1-m}{m} u_{i_{0}}^{-m}\left(t_{0}\right) \delta^{2} u_{i_{0}}\left(t_{0}\right)-K\right) Z_{i_{0}}\left(t_{0}\right)>0 \text { if } 0 \leqslant i_{0} \leqslant I-1, \\
\frac{d}{d t} Z_{i_{0}}(t)>\left(\frac{1-m}{m} u_{I}^{-m}\left(t_{0}\right) \delta^{2} U_{I}\left(t_{0}\right)+\frac{2(1-m+\alpha)}{m h} u_{I}^{\alpha-m}\left(t_{0}\right)-K\right) Z_{I}\left(t_{0}\right)>0 \text { if } i_{0}=I,
\end{aligned}
$$

which is a contradiction with (3.7) and the lemma is completely proved.

Lemma 3.4. Let $\mathrm{U}_{\mathrm{h}}$ be a solution of (2.1)-(2.3) and the initial condition at (2.3) verifies $\delta_{*}^{2} \varphi_{\mathrm{h}} \geqslant 0$ and $\varphi_{i}<\varphi_{i+1}, 0 \leqslant i \leqslant I-1$. Then, $\mathrm{U}_{i}(\mathrm{t})<\mathrm{U}_{i+1}(\mathrm{t}), \quad 0 \leqslant i \leqslant \mathrm{I}-1, \mathrm{t} \in\left[0, \mathrm{~T}_{\mathrm{h}}\right)$.

Proof. We set $Z_{i}(t)=U_{i}(t)-U_{i+1}(t), 0 \leqslant i \leqslant I-1, t \in\left(0, T_{h}\right)$. Let $t_{0}$ be the first $t>0$ such that $Z_{i}(t)<0$ for $t \in\left(0, t_{0}\right)$, but $Z_{i_{0}}\left(t_{0}\right)=0$ for a certain $i_{0} \in\{0, \ldots, I-1\}$. Without lost of generality, we may suppose that $i_{0}$ is the smallest integer which satisfies the above inequality. We have

$$
\frac{d Z_{\mathfrak{i}_{0}}\left(t_{0}\right)}{d t}=\lim _{\epsilon \rightarrow 0} \frac{Z_{\mathfrak{i}_{0}}\left(t_{0}\right)-Z_{\mathfrak{i}_{0}}\left(t_{0}-\epsilon\right)}{\epsilon} \geqslant 0, \quad \delta^{2} Z_{\mathfrak{i}_{0}}\left(t_{0}\right)<0 .
$$

By a simple computation, we obtain

$$
\begin{aligned}
\frac{d Z_{i_{0}}\left(t_{0}\right)}{d t}= & \frac{1}{m} u_{i_{0}+1}^{1-m}\left(t_{0}\right) \delta^{2} Z_{i_{0}}\left(t_{0}\right)+\frac{1}{m}\left(u_{i_{0}}^{1-m}\left(t_{0}\right)-U_{i_{0}+1}^{1-m}\left(t_{0}\right)\right) \delta^{2} u_{i_{0}}\left(t_{0}\right) \text { if } 0 \leqslant i_{0} \leqslant I-2, \\
= & \frac{1}{m} u_{i_{0}+1}^{1-m}\left(t_{0}\right) \delta^{2} Z_{i_{0}}\left(t_{0}\right)<0, \\
\frac{d Z_{I-1}\left(t_{0}\right)}{d t}= & \frac{1}{m} U_{I}^{1-m}\left(t_{0}\right) \delta^{2} Z_{I-1}\left(t_{0}\right)+\frac{1}{m}\left(U_{I-1}^{1-m}\left(t_{0}\right)-U_{I}^{1-m}\left(t_{0}\right)\right) \delta^{2} U_{I-1}\left(t_{0}\right) \\
& -\frac{2}{m h} U_{I}^{1-m+\alpha}\left(t_{0}\right) \text { if } i_{0}=I-1, \\
= & \frac{1}{m} U_{I}^{1-m}\left(t_{0}\right) \delta^{2} Z_{I-1}\left(t_{0}\right)-\frac{2}{m h} U_{I}^{1-m+\alpha}\left(t_{0}\right)<0,
\end{aligned}
$$

which contradict the relation (3.9) and the proof is complete.

Theorem 3.5. Assume that the problem (1.1) has a solution $u \in C^{4,1}\left([0,1] \times\left[0, \mathrm{~T}_{\mathrm{d}}\right]\right)$ and the initial condition at (2.3) verifies

$$
\left\|\varphi_{\mathrm{h}}-\mathrm{u}_{\mathrm{h}}(0)\right\|_{\infty}=\mathrm{o}(1) \text { as } \mathrm{h} \rightarrow 0,
$$

where $\mathfrak{u}_{\mathrm{h}}(\mathrm{t})=\left(\mathrm{u}\left(\mathrm{x}_{0}, \mathrm{t}\right), \ldots, \mathrm{u}\left(\mathrm{x}_{\mathrm{I}}, \mathrm{t}\right)\right)^{\top}$. Then, for $\mathrm{h}$ small enough, the semidiscrete problem (2.1)-(2.3) has a 
unique solution $\mathrm{U}_{\mathrm{h}} \in \mathrm{C}^{1}\left(\left[0, \mathrm{~T}_{\mathrm{d}}\right], \mathbb{R}^{\mathrm{I}+1}\right)$ such that

$$
\max _{0 \leqslant t \leqslant T_{d}}\left\|u_{h}(t)-u_{h}(t)\right\|_{\infty}=\mathrm{O}\left(\left\|\varphi_{h}-u_{h}(0)\right\|_{\infty}+h^{2}\right) \text { as } h \rightarrow 0 .
$$

Proof. Let $\alpha>0$ be such that

$$
\|u(\cdot, t)\|_{\infty} \leqslant \alpha \text { for } t \in\left[0, T_{d}\right] .
$$

Then the problem (2.1)-(2.3) has for each $h$, a unique solution $U_{h} \in C^{1}\left(\left[0, T_{h}\right), \mathbb{R}^{I+1}\right)$. Let $t(h) \leqslant$ $\min \left\{T_{d}, T_{h}\right\}$ be the greatest value of $t>0$ such that

$$
\left\|\mathrm{U}_{\mathrm{h}}(\mathrm{t})-\mathrm{u}_{\mathrm{h}}(\mathrm{t})\right\|_{\infty}<1
$$

The relation (3.10) implies $t(h)>0$ for $h$ small enough. Using the triangle inequality, we obtain

$$
\left\|\mathrm{U}_{h}(\mathrm{t})\right\|_{\infty} \leqslant\|\mathrm{u}(\cdot, \mathrm{t})\|_{\infty}+\left\|\mathrm{U}_{\mathrm{h}}(\mathrm{t})-\mathrm{u}_{\mathrm{h}}(\mathrm{t})\right\|_{\infty} \text { for } \mathrm{t} \in(0, \mathrm{t}(\mathrm{h})),
$$

which implies that

$$
\left\|\mathrm{U}_{\mathrm{h}}(\mathrm{t})\right\|_{\infty}<1+\alpha \text { for } \mathrm{t} \in(0, \mathrm{t}(\mathrm{h})) .
$$

Let $e_{h}(t)=u_{h}(t)-u_{h}(t)$ be the discretization error. Using the Taylor's expansion, we have for $t \in(0, t(h))$,

$$
\begin{aligned}
& \frac{d e_{i}(t)}{d t}-\frac{1}{m} u_{i}^{1-m}(t) \delta^{2} e_{i}(t)=\frac{1-m}{m} \xi_{i}^{-m}(t) \delta^{2} u\left(x_{i}, t\right) e_{i}(t)+O\left(h^{2}\right), \quad 0 \leqslant i \leqslant I-1, \\
& \frac{d e_{I}(t)}{d t}-\frac{1}{m} u_{I}^{1-m}(t) \delta^{2} e_{I}(t)=\left(\frac{1-m}{m} \xi_{I}^{-m}(t) \delta^{2} u\left(x_{I}, t\right)+\frac{2(1-m+\alpha)}{m h} \theta_{I}^{-m+\alpha}(t)\right) e_{I}(t)+O\left(h^{2}\right),
\end{aligned}
$$

where $\xi_{i}(t)$ is an intermediate value between $U_{i}(t)$ and $u\left(x_{i}, t\right)$ for $i \in\{0, \ldots, I\}$ and $\theta_{I}(t)$ the one between $\mathrm{U}_{\mathrm{I}}(\mathrm{t})$ and $\mathrm{u}\left(\mathrm{x}_{\mathrm{I}}, \mathrm{t}\right)$. Using (3.11) and (3.13), there exist $\mathrm{Q}$ and $\mathrm{K}$ positive constants such that

$$
\begin{aligned}
& \frac{d e_{i}(t)}{d t}-\frac{1}{m} U_{i}^{1-m}(t) \delta^{2} e_{i}(t) \leqslant Q\left|e_{i}(t)\right|+K h^{2}, \quad 0 \leqslant i \leqslant I-1, \\
& \frac{d e_{I}(t)}{d t}-\frac{1}{m} U_{I}^{1-m}(t) \delta^{2} e_{I}(t) \leqslant \frac{Q}{h}\left|e_{i}(t)\right|+K h^{2} .
\end{aligned}
$$

Now, we consider the function

$$
Z(x, t)=\left(\left\|\varphi_{h}-u_{h}(0)\right\|_{\infty}+M h^{2}\right) e^{(L+1) t+C x^{2}},
$$

where $M, L, C$ are non-negative constants. We denote by $Z\left(x_{i}, t\right)$ the discretisation in space of $Z(x, t)$. For suitable non-negative constants $M, L, C$, we obtain for $t \in(0, t(h))$,

$$
\begin{aligned}
\frac{d Z\left(x_{i}, t\right)}{d t}-\frac{1}{m} U_{i}^{1-m}(t) \delta^{2} Z\left(x_{i}, t\right) & >Q\left|Z\left(x_{i}, t\right)\right|+K h^{2}, \quad 0 \leqslant i \leqslant I-1, \\
\frac{d Z\left(x_{I}, t\right)}{d t}-\frac{1}{m} U_{I}^{1-m}(t) \delta^{2} Z\left(x_{I}, t\right) & >\frac{Q}{h}\left|Z\left(x_{I}, t\right)\right|+K h^{2}, \\
Z\left(x_{i}, 0\right) & >\left|e_{i}(0)\right| .
\end{aligned}
$$

From Lemma 3.1, we obtain

$$
e_{i}(t)<Z\left(x_{i}, t\right), \quad 0 \leqslant i \leqslant I, t \in(0, t(h)) .
$$

By the same argument, we also prove that

$$
-e_{i}(t)<Z\left(x_{i}, t\right), \quad 0 \leqslant i \leqslant I, t \in(0, t(h)) .
$$


We deduce that

$$
\left\|\mathrm{U}_{\mathrm{h}}(\mathrm{t})-\mathrm{u}_{\mathrm{h}}(\mathrm{t})\right\|_{\infty} \leqslant\left(\left\|\mathrm{U}_{\mathrm{h}}(0)-\mathrm{u}_{\mathrm{h}}(0)\right\|_{\infty}+\mathrm{Mh}^{2}\right) \mathrm{e}^{(\mathrm{L}+1) \mathrm{t}+\mathrm{C}}, \quad \mathrm{t} \in(0, \mathrm{t}(\mathrm{h})) .
$$

To complete the proof of this theorem, we have to show that, for $h$ sufficiently small, $t(h)=T_{d}$. But if it is not true, for some $h$, as small as we like, $t(h)<T_{d}$ and, by (3.12) and (3.14), we obtain

$$
1=\left\|\mathrm{u}_{\mathrm{h}}(\mathrm{t}(\mathrm{h}))-\mathrm{u}_{\mathrm{h}}(\mathrm{t}(\mathrm{h}))\right\|_{\infty} \leqslant\left(\left\|\mathrm{U}_{\mathrm{h}}(0)-\mathrm{u}_{\mathrm{h}}(0)\right\|_{\infty}+M \mathrm{~h}^{2}\right) \mathrm{e}^{(\mathrm{L}+1) \mathrm{T}_{\mathrm{d}}+\mathrm{C}} .
$$

Since the term on the right hand side of the above inequality goes to zero as $h$ tends to zero, we deduce that $1 \leqslant 0$, which is impossible.

\section{Numerical blow-up}

In this section, we prove that the solution $U_{h}$ of the semidiscrete problem (2.1)-(2.3) blows up in finite time and its semidiscrete blow-up time converges to the real one when the mesh size goes to zero.

Theorem 4.1. Let $0<\mathrm{m}<\alpha \leqslant 1$ or $0<\mathrm{m}<1<\alpha$ or $1<\mathrm{m}<\alpha$. Assume that the problem (1.1) has a solution $\mathrm{u}$ which blows up in finite time $\mathrm{T}$ such that $\mathrm{u} \in \mathrm{C}^{4,1}([0,1] \times[0, \mathrm{~T}))$ and the initial condition at (2.3) verifies $\left\|\varphi_{h}-u_{h}(0)\right\|_{\infty}=\mathrm{o}(1)$ as $\mathrm{h} \rightarrow 0$. Then the unique solution $\mathrm{U}_{\mathrm{h}}$ of (2.1)-(2.3) blows up in finite time $\mathrm{T}_{\mathrm{h}}$ for sufficiently small $\mathrm{h}$, and we have the following relation:

$$
\lim _{h \rightarrow 0} T_{h}=T \text {. }
$$

Proof. To prove this theorem, we will use Theorem 1.4 given by Ushijima in [12]. We only have to check three conditions: conditions A0, A1"', and A2' (see [12]).

Step 1 (Condition A0): The solution $u$ of (1.1) blows up in finite time T (see [6]).

Step 2 (Condition A1"'): Let us define functional J as follows :

$$
J[u](t)=\int_{0}^{1} u^{m}(x, t) d x, \quad t \in[0, T) .
$$

We have

$$
\frac{d J(t)}{d t}=\int_{0}^{1}\left(u^{m}\right)_{t}(x, t) d x=\int_{0}^{1} u_{x x}(x, t) d x=u^{\alpha}(1, t) \geqslant \int_{0}^{1} u^{\alpha}(x, t) d x, \geqslant\left(\int_{0}^{1} u^{m}(x, t) d x\right)^{\frac{\alpha}{m}} .
$$

We obtain the last inequality by using Jensen's inequality. Thus, we reach $\frac{d J(t)}{d t} \geqslant(J)^{\alpha / m}$.

Define $H(t)=\frac{1}{2} u^{\alpha}(1, t), t \in[0, T)$. From (4.1), we get $\frac{d}{d t} J(t) \geqslant H(t), t \in[0, T)$ and $\lim _{t \rightarrow T} H(t)=\infty$ since $x=1$ is always a blow-up point.

Now, for $t \in\left[0, T_{h}\right)$ we denote by

$$
\mathrm{J}_{\mathrm{h}}(\mathrm{t})=\mathrm{h} \sum_{\mathrm{i}=0}^{\mathrm{I}} \mathrm{u}_{\mathrm{i}}^{\mathrm{m}}(\mathrm{t}) \quad \text { and } \quad \mathrm{H}_{\mathrm{h}}(\mathrm{t})=\frac{1}{2} \mathrm{u}_{\mathrm{I}}^{\alpha}(\mathrm{t}),
$$

the numerical approximations of $J$ and $H$, respectively. By a simple computation, we obtain for $t \in\left[0, T_{h}\right)$,

$$
\frac{d J_{h}(t)}{d t} \geqslant H_{h}(t) \text { and } \frac{d H_{h}(t)}{d t}=\frac{\alpha}{2} U_{I}^{\alpha-1}(t) \frac{d U_{I}(t)}{d t} \geqslant 0 .
$$

A straightforward calculation yields the following inequality 


$$
\frac{d J_{h}(t)}{d t} \geqslant\left(J_{h}\right)^{\alpha / m}
$$

Putting $G(s)=(s)^{\alpha / m}$, it is clear that

$$
\frac{d J_{h}(t)}{d t} \geqslant G\left(J_{h}\right)
$$

and there exists $s_{0}>0$ such that

$$
\begin{cases}G(s)>0, & \text { for } s>s_{0}, \\ \int_{s_{0}}^{\infty} \frac{d s}{G(s)}<\infty, & \text { since } \frac{\alpha}{m}>1 .\end{cases}
$$

Condition ( $\left.\mathrm{A}^{\prime \prime \prime}\right)$ of theorem 1.4 in [12] is satisfied.

Step 3 (Condition A2'): Using Theorem 3.5, we show that for any $\epsilon>0$,

$$
\lim _{h \rightarrow 0} \sup _{t \in[0, T-\epsilon]}\left|J[u](t)-J_{h}\left[U_{h}\right](t)\right|=0 \text { and } \lim _{h \rightarrow 0} \sup _{t \in[0, T-\epsilon]}\left|H(t)-H_{h}(t)\right|=0 .
$$

Finally, conditions A0, A1"', and A2' are satisfied. According to Theorem 1.4 of [12], we obtain the desired results.

\section{Numerical experiments}

In this section, we estimate the numerical blow-up time of (2.1)-(2.3) by using the algorithm proposed by Hirota and Ozawa [9]. Let $\left\{v_{j}\right\}$ be a geometric sequence. The main idea of this algorithm is to transform the semidiscrete scheme (2.1)-(2.3) into a tractable form by the arc length transformation technique. For each value $v_{j}$, this tractable form is integrated by an ODE solver (here we use DOP54) from 0 to $v_{j}$ in order to generate a linearly convergent sequence to the blow-up time. The sequence is then accelerated by the Aitken $\Delta^{2}$ method. In DOP54, there are three tolerances parameters, AbsTol, RelTol and InitialStep. The parameters AbsTol and RelTol specify the tolerances of the absolute and relative errors, respectively, and InitialStep is used to choose the manner in which the errors are controlled. We set AbsTol $=$ RelTol $=1 . d-15$, InitialStep $=0$, we refer the readers to [7-9] for more details. The sequence $\left\{v_{j}\right\}$ is as follows: $v_{j}=2^{10} \cdot 2^{j}(j=0, \ldots, 12)$ and the initial data

$$
\varphi_{i}=0.5 *(i * h)^{2}+0.5, \quad 0 \leqslant i \leqslant I .
$$

In the rows of the Tables $1-3, T_{h}$ is the approximate blow-up time corresponding to meshes of $I, n$ is the numbers of iterations and the order $s$ of the method is computed from

$$
s=\frac{\log \left(\left(T_{4 h}-T_{2 h}\right) /\left(T_{2 h}-T_{h}\right)\right)}{\log (2)} .
$$

Others illustrations are given by some plots in the Figures 1-3.

\begin{tabular}{llll}
\multicolumn{4}{c}{ Table 1: For $\mathrm{m}=0.5, \alpha=0.9}$. \\
\hline $\mathrm{I}$ & $\mathrm{T}_{\mathrm{h}}$ & $\mathrm{n}$ & $\mathrm{s}$ \\
\hline 16 & 1.116774883 & 63522 & - \\
32 & 1.116616203 & 120086 & - \\
64 & 1.116576512 & 233503 & 2.00 \\
128 & 1.116566594 & 535576 & 2.00 \\
256 & 1.116564124 & 1851197 & 2.00 \\
\hline
\end{tabular}




\begin{tabular}{llll}
\multicolumn{4}{c}{ Table 2: For $m=0.5, \alpha=2}$. \\
\hline $\mathrm{I}$ & $\mathrm{T}_{\mathrm{h}}$ & $\mathrm{n}$ & $\mathrm{s}$ \\
\hline 16 & 0.143979587 & 5441 & - \\
32 & 0.142837935 & 9658 & - \\
64 & 0.142521477 & 17854 & 1.85 \\
128 & 0.142436825 & 33966 & 1.90 \\
256 & 0.142414680 & 66908 & 1.94 \\
512 & 0.142408969 & 153443 & 1.96 \\
1024 & 0.142407511 & 486550 & 1.97 \\
\hline
\end{tabular}

\begin{tabular}{llll}
\multicolumn{5}{c}{ Table 3: For $m=1.1, \alpha=2}$. \\
\hline $\mathrm{I}$ & $\mathrm{T}_{h}$ & $\mathrm{n}$ & $\mathrm{s}$ \\
\hline 16 & 0.367306599 & 5577 & - \\
32 & 0.360220365 & 9375 & - \\
64 & 0.357936256 & 16800 & 1.63 \\
128 & 0.357227601 & 31424 & 1.69 \\
256 & 0.357013541 & 61244 & 1.73 \\
512 & 0.356950138 & 140209 & 1.76 \\
1024 & 0.356931638 & 455441 & 1.78 \\
\hline
\end{tabular}

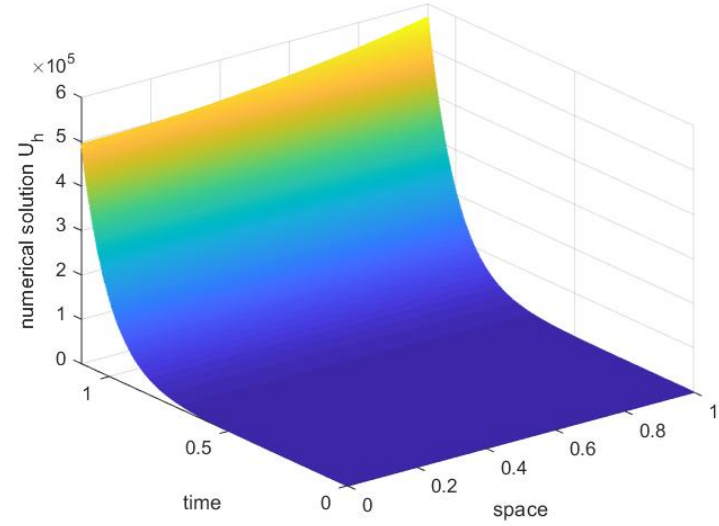

Figure 1: Evolution of the numerical solution for $\mathrm{I}=64, \mathrm{~m}=0.5, \alpha=0.9$ (case where $0<\mathrm{m}<\alpha \leqslant 1$ ).

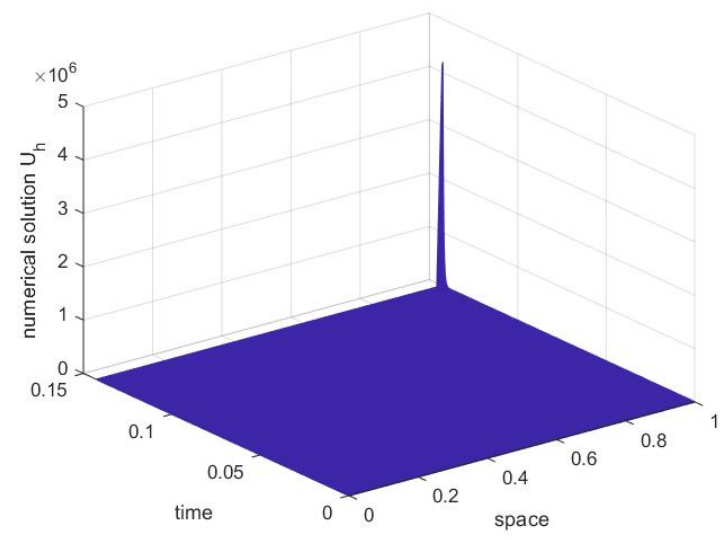

Figure 2: Evolution of the numerical solution for $\mathrm{I}=64, \mathrm{~m}=$ $0.5, \alpha=2$ (case where $0<m<1<\alpha$ ).

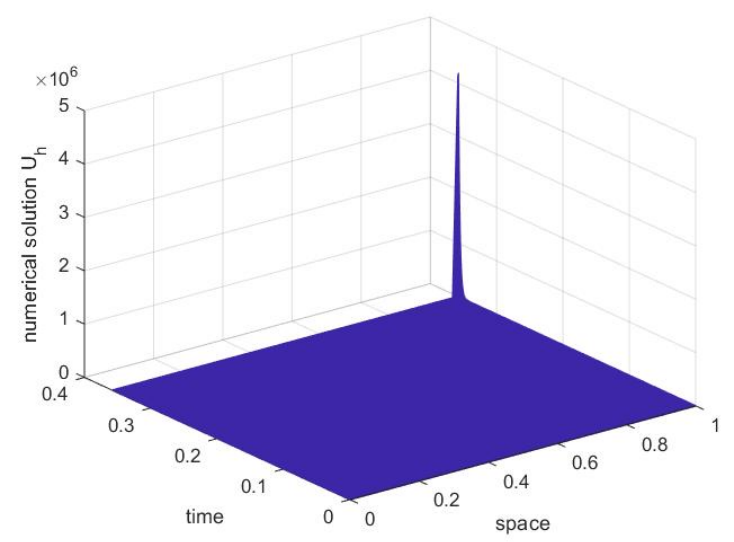

Figure 3: Evolution of the numerical solution for $\mathrm{I}=64, \mathrm{~m}=$ $1.1, \alpha=2$ (case where $1<m<\alpha$ ).

Remark 5.1. We observe from tables above that the growth of $\alpha$ accelerates the blow-up while the growth of $m$ delays it. We also have the convergence of the numerical blow-up time to the continuous one, since the rate of convergence is near 2, which is just the accuracy of the difference approximation in space.

Remark 5.2. Figures 1,2, and 3 show that the numerical solution blows up in finite time on the whole space if $0<m<\alpha \leqslant 1$ and, on the boundary $x=1$ if $0<m<1<\alpha$ or $1<m<\alpha$. Which is in line with the theoretically established result (see [6]). 


\section{References}

[1] K. A. Adou, K. A. Touré, A. Coulibaly, On the numerical quenching time at blow-up, Adv. Math. Sci. J., 8 (2019), 71-85. 1

[2] K. Deng, M. X. Xu, Remarks on blow-up behavior for a nonlinear diffusion equation with neumann boundary conditions, Proc. Amer. Math. Soc., 127 (1999), 167-172. 1

[3] K. B. Edja, K. N'guessan, B. J.-C. Koua, K. A. Touré, Numerical quenching for heat equations with coupled nonlinearboundary flux, Int. J. Anal. Appl., 17 (2019), 1034-1051. 1

[4] R. Ferreira, P. Groisman, J. D. Rossi, Numerical Blow-up for the Porous Medium Equation with a Source, Numer. Methods Partial Differential Equations, 20 (2004), 552-575. 1

[5] R. Ferreira, F. Quirós, The Balance bet we en Nonlinear Inwards and Outwards Boundary Flux for a Nonlinear Heat Equation, J. Differential Equations, 184 (2002), 259-282. 1

[6] J. Filo, Diffusivity versus Absorption through the Boundary, J. Differential Equations, 99 (1992), 281-305. 1, 4, 5.2

[7] A. Ganon, M. M. Taha, A. K. Touré, Blow-up for Semidiscretization of Semilinear Parabolic Equation With Nonlinear Boundary Condition, J. Math. Res., 11 (2019), 1-10. 1, 5

[8] A. Ganon, M. M. Taha, A. K. Touré, Numerical blow-up on whole domain for a quasilinear parabolic equation with nonlinear boundary condition, Adv. Math. Sci. J., 9 (2020), 49-58. 1

[9] C. Hirota, K. Ozawa, Numerical method of estimating the blow-up time and rate of the solution of ordinary differential equations-An application to the blow-up problems of partial differential equations, J. Comput. Appl. Math., 193 (2006), 614-637. 5

[10] Z. X. Jiang, S. Zheng, X. F. Song, Blow-Up Analysis for a Nonlinear Diffusion Equation with Nonlinear Boundary Conditions, Appl. Math. Lett., 17 (2004), 193-199. 1

[11] K. C. N'dri, K. A. Touré, G. Yoro, Numerical blow-up time for a parabolic equation with nonlinear boundary conditions, Int. J. Numer. Methods Appl., 17 (2018), 141-160. 1

[12] T. K. Ushijima, On the Approximation of Blow-up Time for Solutions of Nonlinear Parabolic Equations, Publ. Res. Inst. Math. Sci., 36 (2000), 613-640. 4, 4 